\title{
Terminología del proceso de inteligencia competitiva: estudio teórico y metodológico
}

\author{
Brígida Maria Nogueira Cervantes \\ Mariângela Spotti Lopes Fujita \\ João Batista Ernesto de Moraes \\ Universidade Estadual Paulista - Campus de Marília (Brasil)
}

\section{Resumen}

Se pretende desarrollar un estudio teórico y metodológico del concepto de lenguaje documental y de su interfase con la terminología, verificar la posible aplicación de la metodología del protocolo verbal pensar alto como instrumento de recopilación de términos y formular una propuesta de metodología para la construcción de lenguajes documentales en lo tocante a los procesos de identificación y confirmación de términos. Se utilizan como universo lingüístico los textos científicos y técnicos que tratan del subdominio proceso de inteligencia competitiva, publicados en artículos de revistas y eventos entre 1999 y 2002, incluidos en el proceso de clasificación Qualis de CAPES en categorías indicadoras de calidad con puntuación A en 2002 y de ámbito de circulación nacional. Como resultado se presenta el conjunto de términos identificados y confirmados, con la finalidad de trazar un perfil lingüístico y terminológico de los términos esenciales del subdominio proceso de inteligencia competitiva. Se considera alto el grado de compatibilidad del lenguaje empleado en los textos técnicos y científicos con el utilizado por el usuario. Así, se concluye que la metodología empleada fue eficaz por posibilitar la recopilación del conjunto de términos y permitir el análisis de las manifestaciones de los temas en el momento de la realización de la tarea.

Palabras clave: Lenguaje documental. Terminología. Investigación terminológica temática. Protocolo verbal pensar alto. Proceso de inteligencia competitiva.

\section{Abstract}

The purposes of the present investigation are to develop a theoretical and methodological study of the concept of indexing language and its interface with Terminology, to verify the applicability of the think aloud verbal protocol methodology as a tool for the recollection of terms, and to develop a methodology proposal for the construction of documentary language concerning the processes

Scire. 15 : 2 (jul.-dic. 2009) 83-105. ISSN 1135-3716. 
of identification and confirmation of terms. We use as lingüistic corpus the scientific and technical texts that deal with the sub dominion competitive intelligence process published in journals and conference proceedings between the years of 1999 and 2002 that fit in the classification process Qualis from CAPES in categories which indicated quality with grade A in 2002, nationwide. As a result, the groups of identified and confirmed terms are presented, with the purpose of delineating the linguistic and terminological profile of the essential terms of the sub-dominion competitive intelligence process. The degree of compatibility between the language utilized in technical and scientific texts and the one employed by the user was considered high. Thus, it was concluded that the proposed methodology is effective for collecting the group of terms and enabling the analysis of the subjects in the moment of accomplishing the task.

Keywords: Documentary language. / Terminology. / Thematic terminological research. / Verbal protocol think aloud. / Competitive intelligence process.

\section{Introducción}

La presente investigación tiene como objetivo desarrollar un estudio teóricometodológico del lenguaje documental y de su interfase con la terminología, en la búsqueda de alternativas para el perfeccionamiento de metodologías existentes que orienten la construcción del lenguaje documental en lo tocante al establecimiento de la estructura conceptual, así como en el proceso de identificación y confirmación de términos. Se trata de una investigación aplicada que tiene como objetivo generar conocimientos para la aplicación, práctica y solución de problemas definidos. En cuanto al planteamiento, se caracteriza como un tipo de investigación cualitativa para comprender y clasificar mejor los procesos relativos al tema en estudio.

Los procedimientos metodológicos serán desarrollados con base en la aplicación convergente de la investigación exploratorio-descriptiva, siguiendo las recomendaciones de la terminografía, con la utilización de la metodología de la investigación terminológica temática, observando el conjunto de etapas sugeridas por Rondeau (1984, p. 70).

La metodología del protocolo verbal pensar alto, modalidad interactiva expuesta por Nardi (1999), será utilizada para la forma explícita del proceso de comprensión. La aplicabilidad metodológica se presentará en forma de estudio de caso, utilizando un subdominio - enfocado en el proceso de inteligencia competitiva- del área curricular de Gestión de la Información, en el ámbito del área de la ciencia de la información. Los puntos de interfase de la terminología y el lenguaje documental como instrumento de la ciencia de la información surgen por medio de objetos, actividades, finalidades e instrumentos comunes o similares entre la terminología y la ciencia de la información. En este sentido, se evidencia que la terminología tiene como objeto de uso, teórico y práctico, la lengua de la especialidad y los términos intro- 
ducidos en determinada área. La ciencia de la información también presenta como objeto, entre otros, la lengua de la especialidad y sus vocablos.

A este respecto, Nakayama (1996, p. 74) aclara que los términos "están presentes en el contenido de los documentos, en el lenguaje utilizado (escrito u oral) entre los profesionales de la información, entre los usuarios o entre los profesionales de la información y los usuarios, o incluso entre ellos y la máquina". La autora explica también que "las actividades ejercidas por la terminología y por la ciencia de la información son analizar, investigar, sistematizar y organizar los términos, así como también elaborar obras terminológicas" (ibídem, p. 75). Cabré (1993, 1999), en sus estudios centrados en el área de la terminología, considera que, por ser esta una materia de carácter interdisciplinar, que actúa en distintas especialidades, incluye a los especialistas que hacen uso de ella.

En este sentido, la autora destaca varios tipos de profesionales que necesitan conocimientos de terminología para la elaboración de los trabajos terminológicos; a saber: 1) profesionales del lenguaje (lingüistas teóricos y aplicados, intérpretes, redactores técnicos); 2) especialistas (científicos y técnicos); 3) profesionales de la información y de la documentación; 4) estrategas lingüísticos; y 5) profesionales de la informática especializados en inteligencia artificial. Para la realización del estudio es interesante destacar los tipos de profesionales indicados en los puntos 2 y 3. Cabré (1999, p. 355) argumenta las razones por las que esos profesionales necesitan preparación y graduación en terminología: los especialistas, para participar en trabajos de normalización y para superar las nuevas necesidades terminológicas que puedan surgir en su campo de actuación; los profesionales de la información y de la documentación, para organizar conceptualmente la información, con el fin de que sea recuperada de la forma más ágil y natural posible, y para participar de la elaboración de tesauros y clasificaciones.

De esta forma, se observa que hay puntos de contigüidad entre los propósitos de las dos ciencias: tanto la terminología como la ciencia de la información tienen como finalidad atender a los usuarios en sus necesidades de información. De este modo, ambas poseen diversos instrumentos comunes o similares para la realización de sus actividades, tales como diccionarios, glosarios, enciclopedias, sistemas de clasificación, tesauros, normas, fuentes literarias e informaciones de los profesionales y especialistas del área, entre otros. Todos esos puntos de interfase pueden ser perfeccionados con la aplicación de los principios teóricos y metodológicos de la terminología con vistas a la construcción del lenguaje documental.

\subsection{Terminología, terminografía, teoría del concepto como referencia para la construcción del lenguaje documental}

La terminología posee un objeto propio, el término, signo vehiculador de las designaciones de las lenguas de especialidad, y es considerada un área de aplicación,

Scire. 15 : 2 (jul.-dic. 2009) 83-105. ISSN 1135-3716. 
porque su práctica ha implicado la creación de términos y su análisis, proceso, presentación y uso en la comunicación especializada. De este modo, se resalta la importancia de metodologías que orienten la delimitación de los dominios, la identificación y confirmación de términos, como respuesta al acelerado crecimiento del conocimiento humano, que lleva a la necesidad de incluir nuevas denominaciones. Como práctica, la terminología no se presenta como una innovación, sino que tiene su origen más remoto en el momento en que el hombre sintió la necesidad de dar nombre a sus primeros instrumentos y actividades de trabajo.

La terminología moderna surge en 1931, cuando Eugen Wüster, ingeniero y lingüista austriaco, publica su tesis de doctorado Internationale Sprachnorming in der Technik (Normalización internacional de la terminología técnica), en la que demuestra, principalmente, su preocupación por la cuestión metodológica y normativa. La terminología de Wüster es considerada una herramienta capaz de eliminar las ambigüedades en el ámbito de las comunicaciones científicas y técnicas. Así, este autor contribuye al establecimiento de la teoría general de la terminología (TGT), que desde entonces fundamenta los trabajos terminológicos (Fedor de Diego, 1995, p. 18).

\subsection{Teoría terminológica}

Para Felber y Cabré, estudiosos del área, existen varias acepciones para el vocablo terminología. Felber (1987; ápud Fedor de Diego, 1995) presenta tres conceptos distintos: a) ciencia terminológica o área del conocimiento interdisciplinar y transdisciplinar que trata de los conceptos y de sus representaciones (términos, símbolos y otros); b) conjunto de términos, que representa el sistema de conceptos de un campo especializado; c) publicación, en la cual un sistema de conceptos de un campo especializado está representado por términos. Cabré (1999, p. 18) comenta que reconoce la polisemia del vocablo terminología y relaciona tres nociones diferentes: a) la disciplina, que se ocupa de los términos especializados; b) la práctica, en referencia al conjunto de directrices o principios que rigen la recopilación de los términos; c) el producto generado por esta práctica, es decir, el conjunto de términos de un área especializada.

Se observa que Cabré y Felber dan un carácter autónomo a la terminología: este último, cuando la considera como una ciencia; aquel, cuando admite que la terminología posee principios teóricos y un objeto de estudio definido. Estos estudiosos coinciden en el momento en que se refieren a la terminología como un conjunto de unidades terminológicas pertenecientes a un sistema lingüístico especializado.

En la norma ISO 1087-1 (2000), el vocablo terminología aparece como "un conjunto de términos que representan el sistema de conceptos de un dominio particular”. Esta definición se refiere a la parte aplicada de la terminología. Se en-

Scire. $15: 2$ (jul.-dic. 2009) 83-105. ISSN 1135-3716. 
cuentra, también, en la referida norma, el término ciencia de la terminología, definido como "estudio científico de los conceptos y de los términos en uso en las lenguas de especialidad", de modo que, de acuerdo con Felber, se concede a la terminología la condición de ciencia, esto es, se la somete a todo el rigor que una ciencia requiere. Su principal objeto es la unidad terminológica, o sea, el término como objeto de análisis o como producto para la elaboración de instrumentos terminológicos.

Para Cabré (1999, p. 238), “cada unidad terminológica corresponde a un nudo cognitivo dentro de un campo de especialidad, y el conjunto de esos nudos conectados por relaciones específicas (causa-efecto, todo-parte, contigüidad, anterioridad-posterioridad, y otras) constituye la representación conceptual de dicha especialidad". La terminología está formada por estudios dinámicos, porque se encuentra al servicio de la ciencia y de la tecnología, las cuales se desarrollan con mucha rapidez y provocan que constantemente surjan nuevos conceptos, nuevos términos que precisan ser identificados y confirmados en beneficio del propio progreso de la ciencia y de la tecnología. Cabré (1999, p. 239) enfatiza que "la Terminología, sea cual fuere su temática o el contexto en que se produce, cumple sistemáticamente dos funciones: la de representar el conocimiento especializado, y la de transmitirlo". Así, se supone que la terminología cumple un papel de gran relevancia social, al contribuir a una comunicación más precisa entre especialistas de una determinada área.

\subsection{Terminografía y terminótica}

La terminografía tiene la función de registrar la estandarización para posibilitar una comunicación profesional precisa, buscando la validación en organizaciones y comisiones de normalización y en el parecer de los especialistas. Toda actividad terminológica, desde la identificación de palabras hasta la entrega del producto final, puede realizarse manualmente (Pavel y Nolet, 2002). El desarrollo acelerado de la informática contribuye a la automatización de los trabajos terminológicos. Así, de la vinculación entre terminología e informática se ha originado una nueva área de investigación denominada terminótica. La terminótica o terminología automatizada puede ser entendida como el conjunto de operaciones automatizadas de tratamiento de los términos (Maciel, 2001).

Uno de los requisitos de la terminótica es la elaboración de una ficha terminológica dotada de varios campos que pueden contener información de naturaleza documental, terminológica y lingüística. La ficha terminológica viene a ser el primer paso para la introducción del término en un programa de ordenador. De este modo, se tiene como objeto el tratamiento automático del vocablo, o sea, de la unidad terminológica, y así se forma un componente de la terminografía actual.

Scire. 15 : 2 (jul.-dic. 2009) 83-105. ISSN 1135-3716. 


\subsection{Teoría del concepto}

Ingtraud Dahlberg, estudiosa del área de filosofía, ha posibilitado, con el desarrollo de la teoría del concepto, una base más sólida para la determinación y el entendimiento de lo que se considera concepto, a efectos de representación y recuperación de la información. Según Campos (2001, p. 87), Dahlberg desarrolla esta teoría en el campo de la terminología. En la década de 1970, esta última demuestra la posibilidad de adoptar principios para la elaboración de terminologías en el ámbito de las ciencias sociales (Dahlberg, 1978). En esta misma época, evidencia la ligación entre la Teoría del Concepto y la Teoría de la Clasificación (Dahlberg, 1978a). Posteriormente, utiliza la Teoría del Concepto en el campo de los lenguajes documentales de abordaje alfabético, específicamente, para la elaboración de tesauros (Dahlberg, 1980).

Para Dahlberg (1978, p. 143; ápud Campos, 1994, p. 107), “concepto es unidad de conocimiento, pues conocimiento presupone un entendimiento más objetivo de algo perceptible". De este modo, Dahlberg, al definir el concepto como una unidad de conocimiento que comprende afirmaciones verdaderas sobre un dato, un ítem de referencia representado bajo forma verbal, considera la existencia de tres elementos implicados en la formación del concepto: el referente, las características y la forma verbal.

Todavía según Dahlberg (1978; ápud Campos, 2001, p. 103), el proceso de determinación del concepto tiene lugar en el instante en que un ítem de referencia, un referente, es seleccionado y analizado dentro de un determinado universo. Enseguida se atribuyen predicados al referente escogiendo características relevantes. Estas características deben contribuir al proceso de designación de una forma apropiada, que denote el concepto. Así, el concepto solamente puede ser determinado a partir de la reunión de todos esos elementos que lo componen. La teoría del concepto posibilitó un método para la fijación del contenido del vocablo y para su posicionamiento en un sistema de conceptos.

De acuerdo con la norma ISO 1087-1 (2000), se entiende por sistema de conceptos el "conjunto estructurado de conceptos que tiene como base las relaciones vigentes entre ellos, y en el cual la posición de cada uno está determinada por estas relaciones". El concepto ya no es solo un elemento de significación del vocablo: el término acaba siendo un elemento del propio concepto, el terminum, que sintetiza el concepto como un todo y permite la comunicación. Según Campos (2001), considerar un vocablo como representante de un referente, con sus características, es darle un tratamiento terminológico.

Así, la definición ya no se toma como un recurso auxiliar para minimizar posibles dudas en el uso del término: será incluida en el tesauro como un tipo de nota de aplicación y colocada como un recurso para marcar los límites de la intención del concepto. 


\subsection{La relación entre la terminología y la teoría del concepto en la construcción del lenguaje documental}

De acuerdo con los estudios expuestos anteriormente, se observa que existe la necesidad de una normalización de los términos para establecer la representación y la transferencia de los conocimientos especializados, ya sea para finalidades puramente científicas de la base conceptual como para otras centradas en la fijación y el uso de las lenguas por medio del establecimiento de un registro patrón bien fundamentado. A partir de la exploración teórica interdisciplinar se revela la posibilidad de una investigación de la interfase entre la terminología y la teoría del concepto que concierne a contribuciones teórico-metodológicas para la construcción del lenguaje documental.

Las posibilidades de estudio de esas interfases se encuentran fundamentadas en las indicaciones de varios autores. Wüster (1981, p. 106; ápud Campos, 1995, p. 1), autor de la teoría general de la terminología, observa que hay "semejanza entre las tareas realizadas en la elaboración de un tesauro y en la normalización terminológica en general", y admite que debería existir mayor intercambio entre las áreas. Budin (1993, p. 1; ápud Campos, 1995, p. 3) refuerza esta condición cuando ve la posibilidad de "establecer una teoría de la Terminología que resultara de la unión de las teorías de la ciencia de la información, aplicadas a la construcción y al uso de los lenguajes de documentación (sistemas de clasificación, tesauros, y otros), con la teoría de la organización del conocimiento".

Tálamo et álii (1992, p. 199) enfatizan que es preciso "establecer la interfase entre la terminología y el análisis documental, cuyo estudio debe apoyar de manera creciente la formulación de metodologías para el uso y la elaboración de los lenguajes documentales". Según estos mismos autores, el tesauro solamente ejercerá las funciones de representación y de control terminológico que le son conferidas si viene acompañado de un conjunto de definiciones, por lo menos de los términos específicos del área considerada. Para la determinación del término y de sus relaciones, Campos (2001, p. 100) afirma que "los principios de la Teoría del Concepto se han mostrado útiles para la elaboración de tesauros porque proporcionan bases seguras, tanto para el establecimiento de relaciones como para su realización en el plano verbal, o sea, la determinación de lo que se denomina término".

El tesauro-con-base-en-concepto, según Campos (1994, p. 104), es una "nueva metodología para la elaboración de tesauros", "fundamentada en las cuestiones que envuelven el concepto y las categorías”. Así, Tálamo et álii (1992) adoptan el término tesauro terminológico para esta nueva metodología. Es en este contexto donde actúa la terminología teórica y aplicada, que tiene como objeto de estudio la denominación de los conceptos bajo sus aspectos teóricos y metodológicos, y trata también su representación, sin ambigüedades, en el ámbito de los lenguajes documentales. Su contribución a la lingüística documental se traduce en el 
fundamento teórico y metodológico de una parte importante del conjunto de principios que esta disciplina precisa formalizar como base para establecer los procedimientos adecuados para la construcción de lenguajes documentales.

Tálamo (1997, p. 10) define "lenguaje documental (LD) como un lenguaje construido opuesto al natural; por lo tanto, tiene como objetivo específico encargarse de la información con fines de recuperación". Por este motivo el lenguaje documental actúa como elemento de mediación en el espacio existente entre el emisor y el receptor de la información, de modo que sus actividades convergen en los soportes que la almacenan, como documentos de representación. No hay duda de que la conjunción de estas áreas teóricas, las cuales se suman al área temática que ha de ser estudiada, exige un esfuerzo de investigación. No obstante, el conocimiento que tiene sentido es el generado a partir de la comprensión del contexto del objeto de estudio y de las relaciones e influencias establecidas con otros saberes.

\section{Metodología}

La investigación terminológica temática se presenta en forma de estudio de caso para la delimitación del dominio y el subdominio, la identificación y confirmación de vocablos con vistas a la construcción del lenguaje documental. Se utilizan como universo lingüístico textos científicos y técnicos publicados en artículos de revistas y eventos científicos entre los años 1999 y 2002, relativos al subdominio proceso de inteligencia competitiva. Para el desarrollo de este trabajo terminológico se han seguido las recomendaciones propuestas por la terminografía. De este modo, se tiene como modelo el conjunto de etapas presentado por Rondeau (1984) y complementado, cuando sea necesario, por las orientaciones de Felber (1987) y de la norma ISO 1087-1 (2000).

Cabe mencionar que se ha optado por la modalidad del protocolo verbal interactivo, siguiendo a Nardi (1999), para atender a uno de los objetivos del presente estudio, el de verificar la aplicabilidad de la metodología del protocolo verbal pensar alto como forma de establecimiento de la estructura conceptual (categorización), y para la confirmación de términos en la investigación terminológica, y aun como medio para perfeccionar las metodologías ya existentes. Esa modalidad difiere del protocolo sin interacción por abrir un espacio para el diálogo entre el investigador y el sujeto. Esos procedimientos utilizados, en conjunto, pueden representar una contribución al perfeccionamiento de las metodologías existentes en lo referente al proceso de categorización para la delimitación del dominio y el subdominio, y también para la identificación y confirmación de términos, con vistas a la construcción de lenguajes documentales.

Las etapas necesarias para la realización de la investigación son las siguientes: elección del dominio y de la lengua de trabajo; delimitación del subdominio; consulta a especialistas del subdominio; recopilación del corpus del trabajo ter- 
minológico; establecimiento del árbol del dominio; expansión de la representación del dominio escogido; establecimiento de los límites de la investigación terminológica; recopilación y clasificación de términos; verificación, clasificación y confirmación de términos; y trabajos de presentación de datos terminológicos.

\subsection{Elección del dominio y de la lengua de trabajo}

La elección del dominio para la realización del trabajo terminológico se sitúa en el ámbito del área curricular de Gestión de Información, en el marco de la ciencia de la información. De esta forma, la investigación se realizó para atender especialmente a los intereses terminológicos de los docentes, discípulos y profesionales que actúan en el dominio estudiado. Para la recopilación de los términos se escogió la lengua portuguesa.

\subsection{Delimitación del subdominio}

Según el punto 2.1, el subdominio delimitado para el trabajo terminológico se constituye en el subdominio proceso de inteligencia competitiva, que pertenece al ámbito del área curricular de Gestión de Información, en el marco de la ciencia de la información.

\subsection{Consulta a especialistas del subdominio}

Las frecuentes consultas a los especialistas del subdominio, y también a los investigadores del dominio, han sido fundamentales en el desarrollo de este trabajo terminológico.

\subsection{Recopilación del corpus del trabajo terminológico}

La preocupación por la confiabilidad, representatividad y actualización del trabajo terminológico nos llevó a consultar al especialista del subdominio para someter a su consideración una propuesta de constitución del corpus (Cabré, 1993, p. 278). En dicha propuesta se consideraba que el corpus representativo del subdominio podría estar artículos de revistas científicas y congresos del área de especialidad, publicados en lengua portuguesa entre 1999 y 2002. Para el proceso de elección de los artículos de revistas científicas que formarían parte del corpus del trabajo terminológico se estableció como criterio tomar los artículos identificados en los medios de difusión citados por los programas de posgrado, incluidos en el proceso de clasificación Qualis, concebido por la CAPES, en categorías indicativas de calidad con puntuación A (alta) en el año 2002 y de ámbito de circulación nacional. Entre las revistas científicas del área de la ciencia de la información que ese año obtuvieron puntuación A en el ámbito nacional en el proceso de clasificación Qualis/CAPES, se eligieron para el trabajo los títulos Ciencia de la Información, DataGramaZero, Perspectivas en Ciencia de la Información y Transinformación.

Scire. 15 : 2 (jul.-dic. 2009) 83-105. ISSN 1135-3716. 
Cabe resaltar que la ventaja de la recopilación de términos por medio de las revistas científicas reside en la obtención de términos actualizados según el grado de desarrollo del área, y que son identificados por los pares. Según los estudios de King y Tenopir (1998, p. 176), los artículos de periódicos son leídos con mucha más frecuencia que cualquier otro tipo de publicación, sean revistas comerciales, libros, informes técnicos u otros; y los lectores, que son científicos, buscan en esas lecturas actualización e información para sus investigaciones y para la enseñanza. Se destaca que también fueron recopilados términos en artículos publicados en el Workshop Brasileiro de Inteligência Competitiva e Gestão do Conhecimento, realizados entre los años 1999 y 2001, por tratarse del evento específico del subdominio estudiado. Por lo tanto, el corpus representativo del subdominio contó con un total de 79 artículos.

\subsection{Establecimiento del árbol del dominio}

Con el objetivo de cumplir fielmente el conjunto de etapas del trabajo terminológico señalado por Rondeau (1984, p. 70), el estudio, al ser tratado con el especialista del subdominio, demostró la necesidad de establecer previamente la estructura conceptual o categorización del subdominio estudiado. Surgió también el deseo de probar la aplicación del método del protocolo verbal interactivo con el fin de intentar la categorización. En este sentido, se explicó al investigador del subdominio que esa modalidad del protocolo verbal representaba una innovación, al abrir un espacio para el diálogo entre el investigador y el sujeto durante la realización de la tarea. Se le informó además de que para la realización de la tarea sería necesaria la grabación en casete. Por considerar importante tener puntos concretos de discusión para el desarrollo de la tarea, y con base en los estudios realizados anteriormente, la investigadora esbozó dos propuestas de establecimiento de la estructura conceptual y las presentó al investigador del subdominio. A partir de ese momento se inició el proceso de recopilación del protocolo verbal interactivo, siguiendo a Nardi (1999), con la grabación de los diálogos. A continuación se exponen algunos fragmentos de la grabación a modo de ejemplo.

Fragmentos del protocolo interactivo realizado con el investigador del subdominio para el establecimiento de la estructura conceptual o categorización

\section{Investigadora:}

Según ha podido observar, ¿cuál es el recorte del proceso de inteligencia competitiva en el ámbito de la ciencia de la información? ¿Tendría usted alguna consideración que hacer?

/l

\section{Investigador del subdominio:}

La verdad, yo estaba pensando en el proceso de IC (inteligencia competitiva) en el contexto de la ciencia de la información, es decir, el proceso (proceso de inteligencia competitiva) como un subdominio del área curricular de Gestión de la Información, vinculada al área mayor, la ciencia de la información. 
//

Investigadora:

¿Cree usted que estamos ante de un modo diferente de abordar el proceso de inteligencia competitiva, como un subdominio del área curricular de Gestión de la Información, en el ámbito de la ciencia de la información?

/I

Investigador del subdominio:

¡Exactamente! En el dominio del área curricular Gestión de la Información, en el subdominio proceso de inteligencia competitiva. Solo que, en las categorías, yo colocaría los elementos del proceso de IC.

//

Investigadora:

O sea que con estos arreglos tenemos elementos para elaborar una propuesta de categorización más fundamentada, y que podrá orientar la disposición de los términos en las respectivas categorías.

//

Investigador del subdominio:

Podemos esquematizar de la siguiente manera: como área mayor, la ciencia de la información; como dominio, el área curricular de Gestión de la Información; como subdominio, el proceso de inteligencia competitiva; y como categorías, los elementos que componen el proceso de IC: inteligencia competitiva: conceptos básicos; cultura organizacional; gestión de la información; gestión del conocimiento; innovación tecnológica; instrumentos y tecnologías de información; y profesional que actúa en el proceso de inteligencia competitiva... De repente, puede ser que surja algo nuevo. Hoy, yo no consigo visualizarlo. //

La actuación de la investigadora puede ser considerada como de observadorparticipante, con nivel de participación moderada, porque intentó alternar entre el papel de observadora y el de participante. Cabe resaltar que esa modalidad del protocolo verbal interactivo ha posibilitado el diálogo entre la investigadora y el investigador del subdominio, en el sentido de una interacción en la búsqueda del aprendizaje para la construcción del conocimiento, lo que ha resultado en la elaboración de una nueva propuesta de establecimiento de estructura conceptual o categorización, con el detalle de las categorías que designan aspectos particulares del subdominio proceso de inteligencia competitiva.

El dominio establecido para el trabajo terminológico se constituye en el ámbito del área curricular de Gestión de la Información, dentro de la ciencia de la información, y enfocado en el subdominio proceso de inteligencia competitiva. En este sentido, los elementos que componen el proceso de inteligencia competitiva son cultura organizacional, gestión de la información, gestión del conocimiento, innovación tecnológica, instrumentos y tecnologías de información, inteligencia competitiva: conceptos básicos y profesional que actúa en el proceso de inteligencia competitiva.

Scire. 15 : 2 (jul.-dic. 2009) 83-105. ISSN 1135-3716. 
No obstante, se advierte que se trata de una sugerencia, una propuesta de establecimiento de una estructura conceptual o categorización, para situar el proceso de inteligencia competitiva en el dominio del área curricular de Gestión de la Información, en el ámbito del área de la ciencia de la información, intentando contribuir al proceso de desarrollo terminológico del área curricular de Gestión de la Información. De este modo, se tienen como parámetros las seis áreas curriculares de la ciencia de la información que se establecieron en el $3 .{ }^{\text {er }}$ Encuentro de Directores y $2 .^{\circ}$ de Docentes de Escuelas de Bibliotecología y Ciencia de la Información del Mercosur, que tuvo lugar en Santiago de Chile del 29 al 31 de octubre de 1998.

El acuerdo adoptado entre los países participantes en el evento establece seis áreas curriculares delimitadas de modo que los cursos desarrollen contenidos formativos para el profesional del área, con las respectivas cargas horarias mínimas, tal como se presentan en la tabla I.

\begin{tabular}{|l|c|}
\hline Área curricular & Carga horaria mínima \\
\hline 1. Fundamentos Teóricos de Biblioteconomía y Ciencia de la Información & $14 \%$ \\
\hline 2. Organización y Tratamiento de la Información & $20 \%$ \\
\hline 3. Recursos y Servicios de Información & $20 \%$ \\
\hline 4. Tecnologías de Información & $16 \%$ \\
\hline 5. Gestión de la Información & $20 \%$ \\
\hline 6. Investigación & $10 \%$ \\
\hline
\end{tabular}

Tabla I. Las seis áreas curriculares y sus cargas horarias mínimas (fuente: Acuerdos y recomendaciones, 1998, p. 15).

De esta forma, teniendo como punto de partida la sugerencia de una categorización, en la figura 1 se intenta ilustrar el contexto mediante su representación gráfica. Se expresa a través de una estructura circular para poner de relieve el carácter no necesariamente jerárquico entre las categorías.

Para la organización de los términos recogidos se han considerado como categorías los elementos que componen el proceso de inteligencia competitiva: $\mathrm{cul}$ tura organizacional, gestión de la información, gestión del conocimiento, innovación tecnológica, instrumentos y tecnologías de información, inteligencia competitiva: conceptos básicos y profesional que actúa en el proceso de inteligencia competitiva. Cabe resaltar que esos elementos constituyentes interactúan entre sí y con el propio proceso de inteligencia competitiva. Esta interacción podría resultar en un modelo de relación entre las categorías.

Scire. 15 : 2 (jul.-dic. 2009) 83-105. ISSN 1135-3716. 


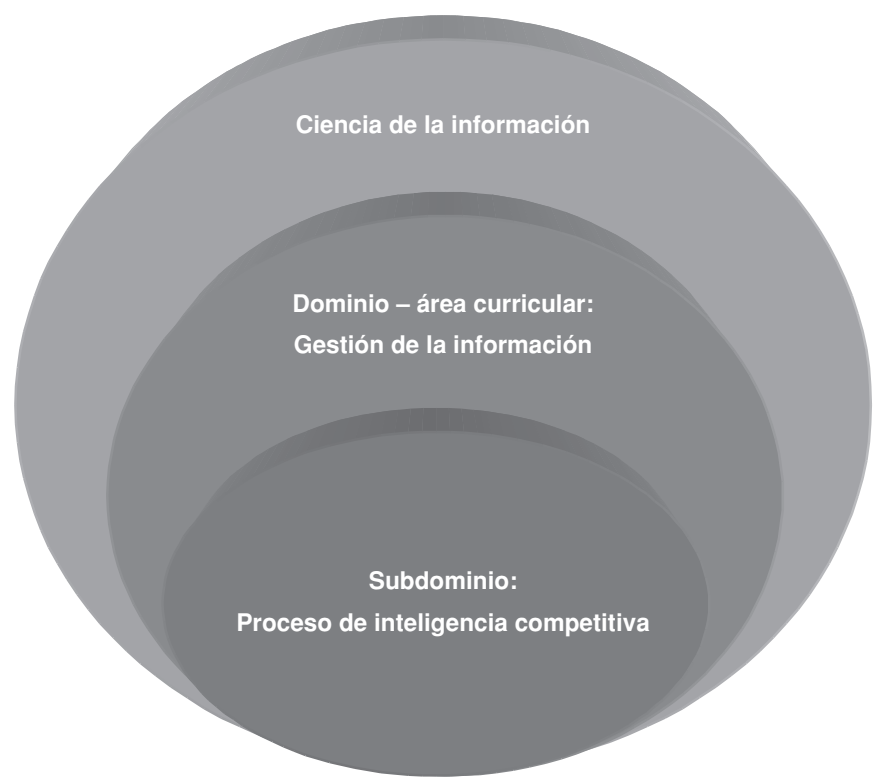

Figura 1. Categorización del proceso de inteligencia competitiva.

\subsection{Expansión de la representación del dominio escogido}

Esta etapa se realizó paralelamente a la anterior, tanto por la orientación de los trabajos para la aplicación del protocolo interactivo —o sea, los preparativos que antecedieron a la aplicación del método, el esbozo de las dos propuestas presentadas para la recopilación de datos - como por la elaboración de la propuesta de establecimiento de la estructura conceptual que guía la investigación terminológica temática.

\subsection{Establecimiento de los límites de la investigación terminológica}

En función de los objetivos propuestos, concretamente el de probar la aplicabilidad de la metodología del protocolo verbal, modalidad interactiva, en conjunto con las demás metodologías ya existentes, se optó por la realización de un sondeo básico de términos. De este modo fueron investigados 79 artículos y recopilados 189 términos. De estos, 181 se confirmaron en el subdominio proceso de inteligencia competitiva. Por este motivo hay que destacar que el universo sondeado permitió completar todas las categorías. Se considera alto el grado de compatibilidad del lenguaje utilizado en los textos estudiados con el empleado por el usuario, aquí representado por el investigador y el profesional del subdominio. Así, como resultado, se pasaron a considerar los 181 términos confirmados por los sujetos participantes en la investigación. 


\subsection{Recopilación y clasificación provisional de términos}

La recopilación de términos fue realizada con base en el universo de documentos establecidos en la etapa de recopilación del corpus de trabajo terminológico (apartado 2.4). Para la realización del trabajo de recopilación de términos se adoptaron como parámetros el subdominio y las categorías establecidas y descritas en la etapa establecimiento del árbol del dominio (apartado 2.5: el subdominio proceso de inteligencia competitiva y las categorías cultura organizacional, gestión de la información, gestión del conocimiento, innovación tecnológica, instrumentos y tecnologías de información, inteligencia competitiva: conceptos básicos y profesional que actúa en el proceso de inteligencia competitiva).

El proceso de recopilación de términos consistió en hacer una lectura de la literatura seleccionado señalando las unidades terminológicas que se encontraban acompañadas de su contexto de uso. El contexto pretende presentar el término que, en este trabajo, se encuentra destacado entre $<>$, en el ámbito de su funcionamiento conceptual. Para el recorte del contexto de aparición se utilizó la recomendación de la norma ISO 1087-1 (2000). Teniendo en cuenta los propósitos de este estudio, y con el objetivo de experimentar todas las etapas, la investigadora actuó también como indizadora para que el conjunto de términos citados realmente correspondiese al corpus propuesto. Los más privilegiados fueron los contextos de aparición informativos, o sea, los contextos definitorios y explicativos, porque se entiende que son los que más datos aportan sobre el significado del término. Cabe destacar que esta opción puede ser recomendable, principalmente, para las áreas que pasan por cambios y que carecen de terminología.

\section{Elaboración de la ficha para la recopilación de términos}

$\mathrm{Al}$ encontrarse con un término, el investigador puede hacer la anotación en una ficha. Existen dos tipos de fichas para esta fase: la de detección y la de citación (Felber, 1987, p. 277). Por juzgarla más adecuada para los propósitos de la investigación, para el registro de los términos se adoptó el tipo de ficha de citación, donde aparece el término en su contexto de aparición. Los términos y contextos detectados eran tecleados directamente en las fichas de citación, al igual que los demás campos existentes. En el momento del registro, el investigador intentaba definir, aunque provisionalmente, la categoría correspondiente al término. Por este motivo las fichas fueron elaboradas por categorías. De hecho, las fichas elaboradas para la realización del trabajo se componen fundamentalmente de planillas electrónicas elaboradas por medio de softwares básicos disponibles en los paquetes de Microsoft, como Word y Excel, entre otros. Aunque la investigación terminológica haya sido realizada de manera tradicional, se buscaron, en la medida de lo posible, los recursos tecnológicos disponibles con vistas a la racionalización del trabajo. Para la estructuración de la ficha de citación utilizada para el registro de los términos 
se consideró que había que rellenar los siguientes campos: categoría — con base en la propuesta de categorización del subdominio—, término-entrada — según aparece en el contexto establecido-, contexto de uso — definición con base en el contexto tal como aparece en la fuente-, sigla de la fuente y número de la página de la cual fueron extraídos el término y el contexto. Al pie de cada ficha constan otros campos: investigador responsable de la recopilación de los términos, fecha de la recopilación del término y, por último, fuente completa, con referencia detallada y con la sigla adoptada entre paréntesis.

\subsection{Verificación y clasificación de la noción/denominación}

Las operaciones efectuadas en esta etapa complementan y mejoran las de la etapa anterior. De este modo, se realizó una conferencia con el fin de identificar posibles fallas ocurridas en el proceso, debidas al tecleo, la ortografía, etcétera, y también para recopilar datos gramaticales. Hay que advertir que, hasta el momento, la investigadora desconoce la existencia de instrumentos terminológicos en el dominio/subdominio estudiado, de modo que para esa conferencia se utilizaron los diccionarios de Dubois (2001) y Houaiss (2001).

\subsection{Confirmación de los términos recopilados para trabajos de presentación de datos terminológicos}

En esta etapa se llevó a cabo el proceso de confirmación de los términos recopilados en sus contextos de aparición, teniéndose en cuenta los criterios de pertinencia y grado de actualización. En esta fase se hizo necesaria la elaboración de la ficha terminológica.

\subsubsection{Ficha terminológica}

Esta ficha fue elaborada de acuerdo con los campos principales propuestos por Rondeau (1984). Todo el contenido de las fichas de citación, utilizado en la etapa de verificación y clasificación de vocablos, fue transferido a la ficha terminológica para la realización del proceso de confirmación de palabras. Las fichas terminológicas presentan el conjunto de términos dispuestos en orden alfabético en las respectivas categorías e incluyen estos campos: término-entrada, contexto de uso, sigla y fuente, 1) preferido — considerado pertinente y bastante recurrente en el subdominio-, 2) no preferido —considerado pertinente, aunque poco utilizado o antiguo-, 3) desconocido - no conocido por el investigador del subdominio o el profesional del subdominio—, 4) rechazado — considerado no pertinente al subdominio-.

2.10.2. Aplicabilidad de la metodología del protocolo verbal pensar alto

La aplicación del protocolo verbal pensar alto como instrumento de recopilación para la confirmación de términos se desarrolló por medio de los procedimientos que se describen a continuación.

Scire. 15 : 2 (jul.-dic. 2009) 83-105. ISSN 1135-3716. 


\subsubsection{Selección de los sujetos}

Para la selección de los sujetos participantes en la investigación se decidió realizar la recopilación de términos con dos categorías de personas, una investigadora y un profesional, pertenecientes al subdominio en estudio.

\subsubsection{Conversación informal con los sujetos}

Se llevó a cabo una conversación informal con cada sujeto de las categorías seleccionadas para invitarlos a participar de la investigación. Se mencionaron los objetivos de estudio y se señaló su importancia para el desarrollo del área. Entonces se concretó la actividad que se iba a realizar y se aclaró que esta consistiría básicamente en la lectura de un documento con el objetivo de confirmar la pertinencia y actualidad de los términos, y que durante toda la lectura sería preciso exteriorizar los procesos mentales. Por este motivo se comunicó a los sujetos que para la realización de la actividad de confirmación de términos sería necesaria la grabación en casete, para lo cual se solicitó su autorización y se informó de que su identidad sería preservada. Se observó una gran motivación en las personas requeridas para participar en el estudio.

\subsubsection{Familiarización con la realización de la tarea}

Antes de la aplicación del protocolo verbal como instrumento de confirmación de los términos se llevó a cabo una actividad destinada a la familiarización con la tarea, utilizando textos con "instrucciones para los sujetos", elaborados con el propósito de presentar los procedimientos para realizar las tareas y, al mismo tiempo, hacer que los sujetos se sintieran cómodos durante la realización de la actividad.

2.10.2.4. Grabación del protocolo verbal pensar alto, modalidad del protocolo interactivo para confirmación de términos

Los protocolos fueron realizados en dos momentos distintos: en primer lugar se procedió a la recopilación con el investigador del subdominio; posteriormente, con el profesional del subdominio aplicándose la técnica del protocolo verbal interactivo para la confirmación de los términos identificados anteriormente por el indizador, según el proceso descrito en la etapa recopilación y clasificación provisional de términos (apartado 2.8). Los protocolos interactivos fueron grabados con el investigador y el profesional del subdominio durante la lectura del texto (ficha terminológica).

Para la grabación del protocolo verbal interactivo con los sujetos, cada uno en una sesión individual de lectura, se informó de que cada uno podría hacer la lectura de forma natural, según su ritmo de estudio o de trabajo, y de que el objetivo era confirmar los términos y las definiciones presentes en la ficha para la confirmación de términos, o sea, verificar si los términos presentes en el texto eran adecuados para representar el subdominio. Se hizo hincapié en que al encontrarse con

Scire. 15 : 2 (jul.-dic. 2009) 83-105. ISSN 1135-3716. 
esos vocablos intentasen exteriorizar los procesos mentales accionados durante la realización de la tarea. De este modo se presentó el texto (ficha terminológica) que contenía los términos y los contextos de aparición identificados en los textos del corpus del trabajo terminológico.

La investigadora puso de relieve que el objetivo de la tarea era confirmar la pertinencia y el grado de actualización de los términos recopilados, y solicitó también que se intentase responder a las cuestiones presentes en la ficha terminológica para la confirmación de los términos. Al principio, la interacción de la investigadora con los sujetos se produjo de forma pasiva, pues actuó como simple observadora mientras los sujetos, al encontrarse con los términos en sus contextos de aparición, exteriorizaban sus procesos mentales de manera fluida. En el transcurso de la recopilación la participación de la investigadora asumió una forma más interactiva. A continuación se presentan como ejemplo algunos fragmentos de la modalidad del protocolo interactivo con el investigador del subdominio y el profesional del subdominio proceso de inteligencia competitiva.

Fragmentos del protocolo interactivo con el investigador del subdominio

Investigador del subdominio:

... En la categoría (inteligencia competitiva - conceptos básicos), con relación al término inteligencia competitiva se presentan tres definiciones. Siendo la primera objetiva, agregando valores a la información, fortaleciendo su carácter estratégico, catalizando así el proceso de crecimiento organizacional. En ese sentido, la recopilación, tratamiento, análisis y contextualización de la información permiten la generación de productos de inteligencia que facilitan y optimizan la toma de decisiones en el ámbito táctico y estratégico. La segunda definición presentada está relacionada con la noción del proceso continuo. Su mayor complejidad está en el hecho de establecer relaciones y conexiones de manera que genere inteligencia para la organización, en la medida en que crea estrategias para escenarios futuros y posibilita la toma de decisiones más segura y asertiva. Y la última (la tercera) trabaja con los dos flujos de información: formales e informales.

/I

¡Qué bien! Aquí todas las definiciones están correctas. Sin embargo, me gusta más la segunda, o sea, [está ligada al concepto de proceso continuo], porque la inteligencia competitiva es un proceso. Su mayor complejidad está en el hecho de establecer relaciones y conexiones, porque la IC se vale de la gestión de la información y de la gestión del conocimiento. En realidad, tiene que establecer relaciones y conexiones con esos dos niveles o ámbitos de gestión para poder ser inteligencia competitiva. ¡Me gusta también eso! Porque a partir del momento en que uno establece relaciones entre lo tácito y lo explícito consigue generar inteligencia para la organización, y, haciendo eso, tiene la posibilidad de ayudar en la estrategia que la organización y las personas van a crear para su actividad, para la propia organización, para la competencia, para la competitividad, y así sucesivamente. En fin, esa segunda definición me agrada porque es más completa.

Scire. 15 : 2 (jul.-dic. 2009) 83-105. ISSN 1135-3716. 
Y la última (la tercera definición) es correcta también, porque de hecho la inteligencia competitiva trabaja tanto en el flujo formal como en el informal. Es correcta, pero demasiado sintética...

//

Investigadora:

En la tercera definición, cuando usted resalta que es muy sintética, ya que es una característica del proceso de inteligencia competitiva, ¿en qué definición podría ser incorporada para que quedase más completa?

//

Investigador del subdominio:

Podría ser incorporada a la (definición) dos, y quedaría de esta forma: [Está relacionada con el concepto del proceso continuo y trabaja esencialmente con los flujos formales e informales. Su mayor complejidad está en el hecho de establecer relaciones y conexiones de forma que genere inteligencia para la organización, en la medida en que crea estrategias para escenarios futuros y posibilita la toma de decisiones de manera más segura y acertada]. Allí cabría perfectamente, y daría una idea más completa que la que ya está en la (definición) dos. //

Fragmentos del protocolo interactivo con el profesional del subdominio

Profesional del subdominio:

... A continuación tenemos la categoría profesional que actúa en el proceso de inteligencia competitiva: redes de analistas, redes de observadores... He notado que no hay entrada para observadores tecnológicos. Es sobre lo que estoy trabajando en el IPT (Instituto de Investigaciones Tecnológicas, São Paulo).

//

Investigadora:

¿Usted tendría una definición para la expresión observadores tecnológicos?

/I

Profesional del subdominio:

En este caso, los observadores tecnológicos tienen la función de verificar el estado de las demandas tecnológicas y de las demandas por innovación, en áreas seleccionadas del sector productivo, por ejemplo: productores de equipos electromédicos, fabricantes de chapas de acero revestido, entre otros. Y seguidamente han de proceder a una serie de actividades volcadas en la evaluación de las competencias de laboratorio para la atención de esas demandas emergentes. Como los observadores precisan estar "con un ojo en el futuro", o sea, pensando en la elaboración de escenarios prospectivos relacionados con esas áreas, la idea básica consiste en formular ayudas para políticas públicas, es decir, para quienes toman decisiones en el ámbito estatal y local, de modo que se pueda llevar a cabo la capacitación técnica y de laboratorio destinada a la atención eficiente de los servicios relacionados con esas demandas. Pienso que es así.

//

Investigadora:

¿Es posible sintetizar esa definición?

/I

Scire. 15 : 2 (jul.-dic. 2009) 83-105. ISSN 1135-3716. 


\section{Profesional del subdominio:}

Podría quedar así: los <observadores tecnológicos> tienen la función de formular ayudas para políticas públicas, o sea, para quienes toman decisiones en el ámbito estatal y local, de modo que se pueda llevar a cabo la capacitación técnica y de laboratorio destinada a la atención eficiente de los servicios relacionados con las demandas emergentes, $\mathrm{o}$ sea, las demandas tecnológicas y las demandas por innovación.

El protocolo interactivo posibilita el diálogo y la interacción del investigador con los sujetos participantes en el estudio. Por eso se ha tenido la oportunidad de interactuar y hacer preguntas durante la realización de las tareas de confirmación de los términos y las definiciones recopiladas. La interacción de la investigadora con los sujetos posibilitó la confirmación del conjunto de los términos recopilados, teniendo en cuenta los criterios de pertinencia y grado de actualización, y además permitió completar las definiciones demasiado sintéticas presentadas en el contexto de uso, teniendo como ejemplo el término inteligencia competitiva, según se señaló en el protocolo del investigador del subdominio. Esta modalidad del protocolo verbal posibilitó también la elaboración de una definición para el término observadores tecnológicos en el momento de la realización de la tarea con el profesional del subdominio. Por este motivo, entre las ventajas de la aplicación de esa técnica de recopilación de datos destaca la posibilidad de hacer preguntas durante la realización de la tarea, con vistas al aprendizaje y a la construcción del conocimiento.

\subsubsection{Transcripción de los protocolos verbales}

Las transcripciones de los protocolos verbales, modalidad interactiva, se realizaron de manera que quedaran reflejadas la comprensión de los sujetos y las estrategias adoptadas para la confirmación de los términos. Para visualizar mejor los procesos adoptados por los sujetos se establecieron las siguientes anotaciones para las transcripciones:

\section{Cursiva}

$\underline{\text { Subrayado }}$

( )

Negrita

Puntos suspensivos

// verbalización del trecho exactamente como aparece en el texto base referencia a un determinado término o expresión comentarios analíticos o descriptivos de la investigadora trecho que mejor ejemplifica el fenómeno en descripción pausas en el habla (sin tener en cuenta su duración)

interrupción de pensamiento/habla y separación de diálogos

(Anotaciones adaptadas de Nardi, 1999, p. 138)

2.10.2.6. Comparación de los términos confirmados por los sujetos

A la finalización del proceso de confirmación de los términos, con base en los paradigmas explicados en el apartado 2.10 .1 y después del análisis de las opciones señaladas en cada categoría, se optó por aislar los términos pertenecientes a los Scire. 15 : 2 (jul.-dic. 2009) 83-105. ISSN 1135-3716. 
campos preferido — término considerado pertinente y de uso regular o frecuentey no preferido - término considerado pertinente pero poco usado-. A continuación se realizó la comparación de los términos confirmados por los sujetos (el investigador y el profesional del subdominio), intentando verificar, por medio del lenguaje utilizado en los textos, el grado de compatibilidad con el lenguaje empleado por los sujetos participantes en la investigación.

\subsubsection{Presentación de los datos terminológicos referentes al subdominio} proceso de inteligencia competitiva y de los elementos que lo componen

De acuerdo con el relato de la investigadora, durante el desarrollo de las etapas de la investigación terminológica temática se verificó que los procedimientos metodológicos propuestos fueron seguidos cuidadosamente. De este modo, se procedió a la estructuración del conjunto de los términos referentes al subdominio proceso de inteligencia competitiva, colocados en orden alfabético en las respectivas categorías. Los términos se encuentran acompañados de su contexto de uso, de la sigla y fuente del documento, y también de los paradigmas preferido y no preferido.

\section{Resultados}

Los resultados obtenidos con la aplicación del instrumento de recopilación de datos protocolo verbal pueden ser observados en el propio desarrollo del estudio del caso, donde se han descrito minuciosamente el proceso de establecimiento de la estructura conceptual o categorización del subdominio y el proceso de confirmación de términos por los sujetos participantes en la investigación.

En la tabla II, donde se presentan los elementos del proceso de inteligencia competitiva, se destaca la categoría más numerosa, o sea, la que presentó mayor cantidad de términos recopilados en el contexto de aparición y que, consecuentemente, fue confirmada por los sujetos participantes en la investigación: instrumentos y tecnologías de la información. De la misma forma, se refleja la categoría que, con

\begin{tabular}{|l|r|}
\hline Categorias & \\
\hline Cultura organizacional & 13 \\
\hline Gestión de la información & 37 \\
\hline Gestión del conocimiento & 23 \\
\hline Innovación tecnológica & 13 \\
\hline Instrumentos y tecnologías de información & 49 \\
\hline Inteligencia competitiva: conceptos básicos & 35 \\
\hline Profesional que actúa en inteligencia competitiva & $\mathbf{1 1}$ \\
\hline Total & 181 \\
\hline
\end{tabular}

Tabla II. Recuento de términos por categorías.

Scire. 15 : 2 (jul.-dic. 2009) 83-105. ISSN 1135-3716. 
base en el corpus investigado, presentó la menor cantidad de términos: profesional que actúa en el proceso de inteligencia competitiva.

Según esos criterios, se optó por la utilización de esas dos categorías para la presentación de los resultados, o sea, del conjunto de términos recopilados en el contexto del subdominio proceso de inteligencia competitiva. Como criterio de análisis se tuvieron en cuenta las categorías con mayor y menor cantidad de términos confirmados por los sujetos participantes en la investigación. Así se verificó que el conjunto de vocablos recopilados y confirmados permitió trazar un perfil terminológico de términos esenciales de dicho subdominio.

\section{Conclusión}

En las páginas precedentes se han expuesto los procedimientos teóricos, metodológicos y prácticos adoptados en la ejecución de un trabajo terminológico temático con los vocablos relacionados con el subdominio referido anteriormente. De este modo se ha justificado la elección del tema, que se debe a cierta carencia de lenguajes mediadores en diversas áreas del conocimiento para fines documentales, principalmente en las áreas que en los últimos años han pasado por cambios y carecen de terminología.

$\mathrm{Al}$ entender la importancia de la construcción de lenguajes documentales compatibles con el lenguaje de búsqueda del usuario del sistema de información de un área especializada, se han fijado los objetivos para el desarrollo de un estudio teórico y metodológico del lenguaje documental y el establecimiento de su interfase con la terminología; se ha verificado la aplicabilidad de la metodología del protocolo verbal pensar alto como instrumento de recopilación de términos y se ha formulado una propuesta de metodología para la construcción del lenguaje documental en lo que se refiere al proceso de identificación y confirmación de términos.

Con base en el desarrollo del estudio de caso y en los resultados obtenidos, se ha comprobado que la metodología propuesta ha contribuido al proceso de aprendizaje, ha posibilitado un mayor contacto con investigadores y profesionales calificados, ha permitido el proceso de aprendizaje con vistas a la construcción del conocimiento y ha fundamentado la formulación de una propuesta para el establecimiento de la estructura conceptual o categorización del subdominio proceso de inteligencia competitiva.

\section{Referencias}

Acuerdos y recomendaciones (1998). // III Encuentro de Directores y II de Docentes de Escuelas de Bibliotecología y Ciencia de la Información del Mercosur (Santiago de Chile, 1998). Anais. Santiago: UTEM, 1998.

Aubert, Francis Henrik (2001). Introdução à metodologia da pesquisa terminológica bilíngüe. São Paulo: Humanitas, 2001, 2. ${ }^{a}$ ed.

Scire. 15 : 2 (jul.-dic. 2009) 83-105. ISSN 1135-3716. 
Barros, Lídia Almeida (2004). Curso básico de terminologia. São Paulo: Edusp, 2004.

Cabré, María Teresa (1993). La terminología: teoría, metodología y aplicaciones. Barcelona: Antártida, 1993.

Cabré, María Teresa (1999). La terminología: representación y comunicación. Barcelona: Institut Universitari de Lingüística Aplicada, 1999.

Campos, Maria Luiza de Almeida (1994). Em busca de princípios comuns na área de representação da informação: uma comparação entre o método de classificação facetada, o método de tesauro-baseado-em-conceito e a teoria geral da terminologia. Disertación de maestría. Río de Janeiro: Universidade Federal do Rio de Janeiro, 1994.

Campos, Maria Luiza de Almeida (1995). Perspectivas para o estudo da área de representação da informação. // Ciência da Informação. 25:2.

Campos, Maria Luiza de Almeida (2001). Linguagem documentária: teorias que fundamentam sua elaboração. Niterói: EdUFF, 2001.

Carvalho, N. (1991). A terminologia técnico-científica: aspectos lingüísticos e metodológicos. Recife: UFPE, 1991.

Dubois, J., et álii. (2001). Dicionário de lingüística. São Paulo: Cultrix, 2001. 8. a ed.

Fedor de Diego, A. (1995). Terminología: teoría y práctica. Caracas: Equinoccio; Unión Latina, 1995.

Felber, H. (1987). Manuel de terminologie. París: Unesco-Infoterm, 1987.

Fujita, Mariângela Spotti Lopes; Nardi, Maria Isabel Asperti; Fagundes, Silvana Aparecida (2003). A observação da leitura documentária por meio de Protocolo Verbal. // Rodrigues, Georgete Medleg Rodrigues; Lopes, Ilza Leite (orgs.). Organização e representação do conhecimento na perspectiva da Ciência da Informação. Brasilia: Thesaurus, 2003.

Fujita, Mariângela Spotti Lopes; Nardi, Maria Isabel Asperti; Fagundes, Silvana Aparecida (1999). A leitura do indexador: estudo de observação. // Perspectivas em Ciência da Informação. 4:1 (en.-jun. 1999) 101-116.

Houaiss, A. (2001). Dicionário eletrônico Houaiss da língua portuguesa. Río de Janeiro: Objetiva, 2001. CD-ROM.

ISO 1087-1 (2000). Terminology work - Vocabulary - Part 1: Theory and application. [Ginebra]: ISO, 2000.

King, D. W.; Tenopir, C. (1998). A publicação de revistas eletrônicas: economia da produção, distribuição e uso. // Ciência da Informação. 27:2 (1998) 176-182.

Maciel, A. M. B. (2001). TERMISUL e terminótica. // Krieger, M. da G.; Maciel, A. M. B. (orgs.). Temas de terminologia. Porto Alegre; São Paulo: Universidade / UFRGS; Humanitas / USP, 2001. 344-363.

Nakayama, H. (1996). Terminologia aplicada à ciência da informação: da produção de vocabulário técnico-científico bilíngüe (japonês-português) na área do ensino da língua japonesa. Tesis de doctorado. São Paulo: Universidade de São Paulo, 1996.

Nardi, M. I. A. (1999). A metáfora e a prática de leitura como evento social: instrumentos do pensar a biblioteconomia do futuro. Tesis de doctorado. São Paulo: Pontifícia Universidade Católica, 1999.

Scire. 15 : 2 (jul.-dic. 2009) 83-105. ISSN 1135-3716. 
Pavel, S.; Nolet, D. (2002). Manual de terminología. Quebec: Ministerio de Obras Públicas y Servicios Gubernamentales de Canadá, 2002.

Rondeau, G. (1984). Introduction à la terminologie. Québec: Gaëtan Morin, 1984.

Tálamo, M. de F. G. M. (1997). Linguagem documentária. São Paulo: APB (“Ensaios APB”, 45), 1997.

Tálamo, M. de F. G. M., et álii (1992). Contribuição da terminologia para a elaboração de tesauros. // Ciência da Informação. 21:3 (set.-dez. 1992) 197-200.

Recibido: 2005-10-10. Revisado: 2006-03-10. Aceptado: 2008-12-07

Scire. 15 : 2 (jul.-dic. 2009) 83-105. ISSN 1135-3716. 